\title{
Almost increasing sequences and their new applications
}

Hüseyin Bor ${ }^{*}$

"Correspondence:

hbor33@gmail.com

P.O. Box 121, Bahçelievler, Ankara 06502, Turkey

\begin{abstract}
In this paper, we generalize a known theorem dealing with $|C, 1|_{k}$ summability factors to the $|C, \alpha|_{k}$ summability factors of infinite series using an almost increasing sequence. This theorem also includes some known and new results.
\end{abstract}

MSC: 26D15; 40D15; 40F05; 40G05

Keywords: increasing sequences; Cesàro mean; summability factors; Hölder inequality; Minkowski inequality

\section{Introduction}

A positive sequence $\left(b_{n}\right)$ is said to be an almost increasing sequence if there exists a positive increasing sequence $\left(c_{n}\right)$ and two positive constants $A$ and $B$ such that $A c_{n} \leq b_{n} \leq B c_{n}$ (see [1]). Let $\sum a_{n}$ be a given infinite series with the sequence of partial sums $\left(s_{n}\right)$. By $t_{n}^{\alpha}$ we denote the $n$th Cesàro mean of order $\alpha$, with $\alpha>-1$, of the sequence $\left(n a_{n}\right)$, that is,

$$
t_{n}^{\alpha}=\frac{1}{A_{n}^{\alpha}} \sum_{v=0}^{n} A_{n-\nu}^{\alpha-1} v a_{v}
$$

where

$$
A_{n}^{\alpha}=\left(\begin{array}{c}
n+\alpha \\
n
\end{array}\right)=\frac{(\alpha+1)(\alpha+2) \cdots(\alpha+n)}{n !}=O\left(n^{\alpha}\right), \quad A_{-n}^{\alpha}=0 \quad \text { for } n>0 .
$$

The series $\sum a_{n}$ is said to be summable $|C, \alpha|_{k}, k \geq 1$, if (see [2])

$$
\sum_{n=1}^{\infty} \frac{1}{n}\left|t_{n}^{\alpha}\right|^{k}<\infty
$$

If we take $\alpha=1$, then $|C, \alpha|_{k}$ summability reduces to $|C, 1|_{k}$ summability.

\section{Known result}

Many works dealing with an application of almost increasing sequences to the absolute Cesàro summability factors of infinite series have been done (see [3-11]). Among them, in [10], the following main theorem dealing with $|C, 1|_{k}$ summability factors has been proved.

๑ 2013 Bor; licensee Springer. This is an Open Access article distributed under the terms of the Creative Commons Attribution License (http://creativecommons.org/licenses/by/2.0), which permits unrestricted use, distribution, and reproduction in any medium, provided the original work is properly cited. 
Theorem A Let $\left(\varphi_{n}\right)$ be a positive sequence and $\left(X_{n}\right)$ be an almost increasing sequence. If the conditions

$$
\begin{aligned}
& \sum_{n=1}^{\infty} n\left|\Delta^{2} \lambda_{n}\right| X_{n}<\infty, \\
& \left|\lambda_{n}\right| X_{n}=O(1) \quad \text { as } n \rightarrow \infty, \\
& \varphi_{n}=O(1) \quad \text { as } n \rightarrow \infty, \\
& n \Delta \varphi_{n}=O(1) \quad \text { as } n \rightarrow \infty, \\
& \sum_{v=1}^{n} \frac{\left|t_{v}\right|^{k}}{v X_{v}^{k-1}}=O\left(X_{n}\right) \quad \text { as } n \rightarrow \infty
\end{aligned}
$$

are satisfied, then the series $\sum a_{n} \lambda_{n} \varphi_{n}$ is summable $|C, 1|_{k}, k \geq 1$.

\section{The main result}

The aim of this paper is to generalize Theorem $\mathrm{A}$ to the $|C, \alpha|_{k}$ summability in the following form.

Theorem Let $\left(\varphi_{n}\right)$ be a positive sequence and let $\left(X_{n}\right)$ be an almost increasing sequence.

If the conditions (4), (5), (6) and (7) are satisfied, and the sequence $\left(w_{n}^{\alpha}\right)$ defined by (see [12])

$$
w_{n}^{\alpha}= \begin{cases}\left|t_{n}^{\alpha}\right|, & \alpha=1, \\ \max _{1 \leq v \leq n}\left|t_{v}^{\alpha}\right|, & 0<\alpha<1,\end{cases}
$$

satisfies the condition

$$
\sum_{v=1}^{n} \frac{\left(w_{v}^{\alpha}\right)^{k}}{v X_{v}^{k-1}}=O\left(X_{n}\right) \quad \text { as } n \rightarrow \infty
$$

then the series $\sum a_{n} \lambda_{n} \varphi_{n}$ is summable $|C, \alpha|_{k}, 0<\alpha \leq 1,(\alpha-1) k>-1$ and $k \geq 1$.

Remark It should be noted that if we take $\alpha=1$, then we get Theorem A. In this case, condition (10) reduces to condition (8) and the condition ' $(\alpha-1) k>-1$ ' is trivial.

We need the following lemmas for the proof of our theorem.

Lemma 1 [13] If $0<\alpha \leq 1$ and $1 \leq v \leq n$, then

$$
\left|\sum_{p=0}^{v} A_{n-p}^{\alpha-1} a_{p}\right| \leq \max _{1 \leq m \leq \nu}\left|\sum_{p=0}^{m} A_{m-p}^{\alpha-1} a_{p}\right| .
$$

Lemma 2 [14] Under the conditions (4) and (5), we have

$$
\begin{aligned}
& n X_{n}\left|\Delta \lambda_{n}\right|=O(1) \quad \text { as } n \rightarrow \infty, \\
& \sum_{n=1}^{\infty} X_{n}\left|\Delta \lambda_{n}\right|<\infty .
\end{aligned}
$$




\section{Proof of the Theorem}

Let $\left(T_{n}^{\alpha}\right)$ be the $n$th $(C, \alpha)$ mean, with $0<\alpha \leq 1$, of the sequence $\left(n a_{n} \lambda_{n} \varphi_{n}\right)$.

Then, by (1), we find that

$$
T_{n}^{\alpha}=\frac{1}{A_{n}^{\alpha}} \sum_{v=1}^{n} A_{n-\nu}^{\alpha-1} v a_{\nu} \lambda_{v} \varphi_{n}
$$

Thus, applying Abel's transformation first and then using Lemma 1, we have that

$$
\begin{aligned}
T_{n}^{\alpha}= & \frac{1}{A_{n}^{\alpha}} \sum_{v=1}^{n-1} \Delta\left(\lambda_{v} \varphi_{n}\right) \sum_{p=1}^{v} A_{n-p}^{\alpha-1} p a_{p}+\frac{\lambda_{n} \varphi_{n}}{A_{n}^{\alpha}} \sum_{v=1}^{n} A_{n-v}^{\alpha-1} v a_{v} \\
= & \frac{1}{A_{n}^{\alpha}} \sum_{v=1}^{n-1}\left(\lambda_{v} \Delta \varphi_{v}+\varphi_{v+1} \Delta \lambda_{v}\right) \sum_{p=1}^{v} A_{n-p}^{\alpha-1} p a_{p}+\frac{\lambda_{n} \varphi_{n}}{A_{n}^{\alpha}} \sum_{v=1}^{n} A_{n-v}^{\alpha-1} v a_{v}, \\
\left|T_{n}^{\alpha}\right| \leq & \frac{1}{A_{n}^{\alpha}} \sum_{v=1}^{n-1}\left|\lambda_{v} \Delta \varphi_{v}\right|\left|\sum_{p=1}^{v} A_{n-p}^{\alpha-1} p a_{p}\right|+\frac{1}{A_{n}^{\alpha}} \sum_{v=1}^{n-1}\left|\varphi_{v+1} \Delta \lambda_{v}\right|\left|\sum_{p=1}^{v} A_{n-p}^{\alpha-1} p a_{p}\right| \\
& +\frac{\left|\lambda_{n} \varphi_{n}\right|}{A_{n}^{\alpha}}\left|\sum_{v=1}^{v} A_{n-v}^{\alpha-1} v a_{v}\right| \\
\leq & \frac{1}{A_{n}^{\alpha}} \sum_{v=1}^{n-1} A_{v}^{\alpha} w_{v}^{\alpha}\left|\lambda_{v}\right|\left|\Delta \varphi_{v}\right|+\frac{1}{A_{n}^{\alpha}} \sum_{v=1}^{n-1} A_{v}^{\alpha} w_{v}^{\alpha}\left|\varphi_{v+1}\right|\left|\Delta \lambda_{v}\right|+\left|\lambda_{n}\right|\left|\varphi_{n}\right| w_{n}^{\alpha} \\
= & T_{n, 1}^{\alpha}+T_{n, 2}^{\alpha}+T_{n, 3}^{\alpha} .
\end{aligned}
$$

To complete the proof of the theorem, by Minkowski's inequality, it is sufficient to show that

$$
\sum_{n=1}^{\infty} n^{-1}\left|T_{n, r}^{\alpha}\right|^{k}<\infty \quad \text { for } r=1,2,3
$$

Now, when $k>1$, applying Hölder's inequality with indices $\mathrm{k}$ and $k^{\prime}$, where $\frac{1}{k}+\frac{1}{k^{\prime}}=1$, we get that

$$
\begin{aligned}
\sum_{n=2}^{m+1} n^{-1}\left|T_{n, 1}^{\alpha}\right|^{k} & \leq \sum_{n=2}^{m+1} n^{-1}\left(A_{n}^{\alpha}\right)^{-k}\left\{\sum_{v=1}^{n-1} A_{v}^{\alpha} w_{v}^{\alpha}\left|\Delta \varphi_{v}\right|\left|\lambda_{v}\right|\right\}^{k} \\
& =O(1) \sum_{n=2}^{m+1} \frac{1}{n^{1+\alpha k}} \sum_{v=1}^{n-1}\left(v^{\alpha}\right)^{k}\left(w_{v}^{\alpha}\right)^{k}\left|\Delta \varphi_{v}\right|^{k}\left|\lambda_{v}\right|^{k}\left\{\sum_{v=1}^{n-1} 1\right\}^{k-1} \\
& =O(1) \sum_{n=2}^{m+1} \frac{1}{n^{2+(\alpha-1) k}} \sum_{v=1}^{n-1} v^{\alpha k}\left(w_{v}^{\alpha}\right)^{k}\left|\lambda_{v}\right|^{k} \frac{1}{v^{k}} \\
& =O(1) \sum_{v=1}^{m} v^{\alpha k}\left(w_{v}^{\alpha}\right)^{k} v^{-k}\left|\lambda_{v}\right|^{k} \sum_{n=v+1}^{m+1} \frac{1}{n^{2+(\alpha-1) k}} \\
& =O(1) \sum_{v=1}^{m} v^{\alpha k}\left(w_{v}^{\alpha}\right)^{k} v^{-k}\left|\lambda_{v}\right|^{k} \int_{v}^{\infty} \frac{d x}{x^{2+(\alpha-1) k}}
\end{aligned}
$$




$$
\begin{aligned}
& =O(1) \sum_{v=1}^{m}\left(w_{v}^{\alpha}\right)^{k}\left|\lambda_{v}\right|\left|\lambda_{v}\right|^{k-1} \frac{1}{v} \\
& =O(1) \sum_{v=1}^{m}\left(w_{v}^{\alpha}\right)^{k}\left|\lambda_{v}\right| \frac{1}{v X_{v}^{k-1}} \\
& =O(1) \sum_{v=1}^{m-1} \Delta\left|\lambda_{v}\right| \sum_{r=1}^{v} \frac{\left(w_{r}^{\alpha}\right)^{k}}{r X_{r}^{k-1}}+O(1)\left|\lambda_{m}\right| \sum_{v=1}^{m} \frac{\left(w_{v}^{\alpha}\right)^{k}}{v X_{v}^{k-1}} \\
& =O(1) \sum_{v=1}^{m}\left|\Delta \lambda_{v}\right| X_{v}+O(1)\left|\lambda_{m}\right| X_{m}=O(1) \quad \text { as } m \rightarrow \infty
\end{aligned}
$$

by virtue of the hypotheses of the theorem and Lemma 2 . Again, we get that

$$
\begin{aligned}
\sum_{n=2}^{m+1} n^{-1}\left|T_{n, 2}^{\alpha}\right|^{k} & \leq \sum_{n=2}^{m+1} n^{-1}\left(A_{n}^{\alpha}\right)^{-k}\left\{\sum_{v=1}^{n-1} A_{v}^{\alpha} w_{v}^{\alpha}\left|\varphi_{v+1}\right|\left|\Delta \lambda_{v}\right|\right\}^{k} \\
& =O(1) \sum_{n=2}^{m+1} \frac{1}{n^{1+\alpha k}}\left\{\sum_{v=1}^{n} v^{\alpha}\left(w_{v}^{\alpha}\right)\left|\Delta \lambda_{v}\right|\right\}^{k} \\
& =O(1) \sum_{n=2}^{m+1} \frac{1}{n^{1+\alpha k}} \sum_{v=1}^{n-1} v^{\alpha k}\left(w_{v}^{\alpha}\right)^{k}\left|\Delta \lambda_{v}\right|^{k}\left\{\sum_{v=1}^{n-1} 1\right\}^{k-1} \\
& =O(1) \sum_{n=2}^{m+1} \frac{1}{n^{2+(\alpha-1) k}} \sum_{v=1}^{n-1} v^{\alpha k}\left(w_{v}^{\alpha}\right)^{k}\left|\Delta \lambda_{v}\right|^{k} \\
& =O(1) \sum_{v=1}^{m} v^{\alpha k}\left(w_{v}^{\alpha}\right)^{k}\left|\Delta \lambda_{v}\right|\left|\Delta \lambda_{v}\right|^{k-1} \sum_{n=v+1}^{m+1} \frac{1}{n^{2+(\alpha-1) k}} \\
& =O(1) \sum_{v=1}^{m} \frac{v^{\alpha k}\left(w_{v}^{\alpha}\right)^{k}\left|\Delta \lambda_{v}\right|}{v^{k-1} X_{v}^{k-1}} \int_{v}^{\infty} \frac{d x}{x^{2+(\alpha-1) k}} \\
& =O(1) \sum_{v=1}^{m} v\left|\Delta \lambda_{v}\right| \frac{\left(w_{v}^{\alpha}\right)^{k}}{v X_{v}^{k-1}} \\
& =O(1) \sum_{v=1}^{m} \Delta\left(v\left|\Delta \lambda_{v}\right|\right) \sum_{r=1}^{v} \frac{\left(w_{r}^{\alpha}\right)^{k}}{r X_{r}^{k-1}}+O(1) m\left|\Delta \lambda_{m}\right| \sum_{v=1}^{m} \frac{\left(w_{v}^{\alpha}\right)^{k}}{v X_{v}^{k-1}} \\
& =O(1) \sum_{v=1}^{m-1} v\left|\Delta^{2} \lambda_{v}\right| X_{v}+O(1) \sum_{v=1}^{m-1} X_{v}\left|\Delta \lambda_{v}\right|+O(1) m\left|\Delta \lambda_{m}\right| X_{m} \\
& =O(1) \operatorname{as}_{m} m \rightarrow \infty
\end{aligned}
$$

by hypotheses of the theorem and Lemma 2 . Finally, as in $T_{n, 1}^{\alpha}$, we have that

$$
\begin{aligned}
\sum_{n=1}^{m} n^{-1}\left|T_{n, 3}^{\alpha}\right|^{k} & =\sum_{n=1}^{m} n^{-1}\left|\lambda_{n} \varphi_{n} w_{n}^{\alpha}\right|^{k} \\
& =O(1) \sum_{n=1}^{m} \frac{\left(w_{n}^{\alpha}\right)^{k}\left|\lambda_{n}\right|}{n X_{n}^{k-1}}=O(1) \quad \text { as } m \rightarrow \infty
\end{aligned}
$$


by virtue of the hypotheses of the theorem and Lemma 2 . This completes the proof of the theorem. Also, if we take $k=1$, then we get a new result concerning the $|C, \alpha|$ summability factors of infinite series.

\section{Competing interests}

The author declares that he has no competing interests.

\section{Acknowledgements}

Dedicated to Professor Hari M Srivastava.

The author expresses his thanks to the referees for their useful comments and suggestions.

Received: 9 January 2013 Accepted: 12 April 2013 Published: 25 April 2013

\section{References}

1. Bari, NK, Stečkin, SB: Best approximation and differential properties of two conjugate functions. Tr. Mosk. Mat. Obŝ. 5 , 483-522 (1956) (in Russian)

2. Flett, TM: On an extension of absolute summability and some theorems of Littlewood and Paley. Proc. Lond. Math. Soc. 7, 113-141 (1957)

3. Bor, H: An application of almost increasing sequences. Math. Inequal. Appl. 5(1), 79-83 (2002)

4. Bor, H, Srivastava, HM: Almost increasing sequences and their applications. Int. J. Pure Appl. Math. 3, 29-35 (2002)

5. Bor, H: A study on almost increasing sequences. JIPAM. J. Inequal. Pure Appl. Math. 4(5), Article ID 97 (2003)

6. Bor, H, Leindler, L: A note on $\delta$-quasi-monotone and almost increasing sequences. Math. Inequal. Appl. 8(1), 129-134 (2005)

7. Bor, H, Özarslan, HS: On the quasi-monotone and almost increasing sequences. J. Math. Inequal. 1(4), 529-534 (2007)

8. Bor, H: An application of almost increasing sequences. Appl. Math. Lett. 24(3), 298-301 (2011)

9. Bor, H: On a new application of almost increasing sequences. Math. Comput. Model. 53(1-2), 230-233 (2011)

10. Sulaiman, WT: On a new application of almost increasing sequences. Bull. Math. Anal. Appl. 4(3), 29-33 (2012)

11. Bor, H, Srivastava, HM, Sulaiman, WT: A new application of certain generalized power increasing sequences. Filomat 26(4), 871-879 (2012)

12. Pati, T: The summability factors of infinite series. Duke Math. J. 21, 271-284 (1954)

13. Bosanquet, LS: A mean value theorem. J. Lond. Math. Soc. 16, 146-148 (1941)

14. Mazhar, SM: Absolute summability factors of infinite series. Kyungpook Math. J. 39, 67-73 (1999)

doi:10.1186/1029-242X-2013-207

Cite this article as: Bor: Almost increasing sequences and their new applications. Journal of Inequalities and Applications 2013 2013:207.

\section{Submit your manuscript to a SpringerOpen ${ }^{\circ}$ journal and benefit from:}

- Convenient online submission

Rigorous peer review

- Immediate publication on acceptance

- Open access: articles freely available online

- High visibility within the field

- Retaining the copyright to your article 